\title{
Microscope or MACROscope - Which System Provides a Better Scope on Image Analysis??
}

\author{
J. Woo*, T. Nicklee*, D.W. Hedley*, and P. Constantinou* \\ *Ontario Cancer Institute/Princess Margaret Hospital, Toronto, Ontario, M5G 2M9
}

Quantitative analysis of multi fluorescence labeled whole tumour cut sections can provide invaluable information on molecular relationships. Our group has a particular interest in the biology of tumour hypoxia, since this is a bad prognosis feature in human cancers due to the activation of genes involved in therapy resistance and metastasis formation. Cervical carcinoma cell lines ME180 and SiHa were grown as xenografts in mice. Frozen tissue sections were cut from these and human cervical carcinoma biopsies. They were stained with the hypoxia markers HIF-1 $\alpha-C y 3$, CA9-Alexa 488 and the vascular marker CD31-Cy5. Images of the multi stained whole tissue sections were captured using fluorescence microscopy and a novel laser scanning MACROscope [1].

An Olympus fluorescence microscope with a mercury arc burner, LEP high precision motorized microscope stage, and a Quantix cooled CCD camera was used. The system was controlled by MicroComputer Imaging Device Elite software (Imaging Research, St Catharine's, Ontario). The Elite software allows the entire tissue section to be acquired field-by-field, stitching the images together to create one "tiled" image. Using a 10x objective it took approximately 15 minutes to tile each fluorochrome of a tissue section $7 \mathrm{~mm}$ in diameter. The resulting image files were large, but allowed resolution to be maintained when zooming in on nuclear detail.

The MACROscope was developed at the University of Waterloo, Ontario. It is a three colour laser confocal scanning system with a sophisticated objective lens hat allows distortion-free imaging of areas up to $2 \mathrm{~cm}^{2}$. The excitation wavelengths correspond to those of Alexa 488 (blue light) Cy3 (green light) and Cy5 (red light). It took the laser scanning MACROscope about 3 minutes per fluorochrome to acquire theentire tissue section. The image files were small and when these images were zoomed, they became pixilated before nuclear detail could be resolved.

Both fluorescence microscopy and the MACROscope are useful tools for acquiring large images of whole cut tissue sections. In addition to tumour hypoxia, analysis of the spatial relationships between tissue architecture and the expression of multiple molecular markers defined by immuofluorescence or in situ hybridization is likely to provide a powerful technique in cancer research and molecular diagnosis. The molecular markers of interest will dictate which system is best utilized. The fluorescence microscope is efficient for smaller tissue sections or when resolving fine detail is important. The MACROscope is advantageous when a large number of whole cut tissue section images are required and fine detail is not required.

\section{References}

[1] P. Constantinou et al, Journal of Biomedical Optics 6(3) (July 2001) 326.

[2] This work was supported by the Canadian Institute for Photonics Innovation. 


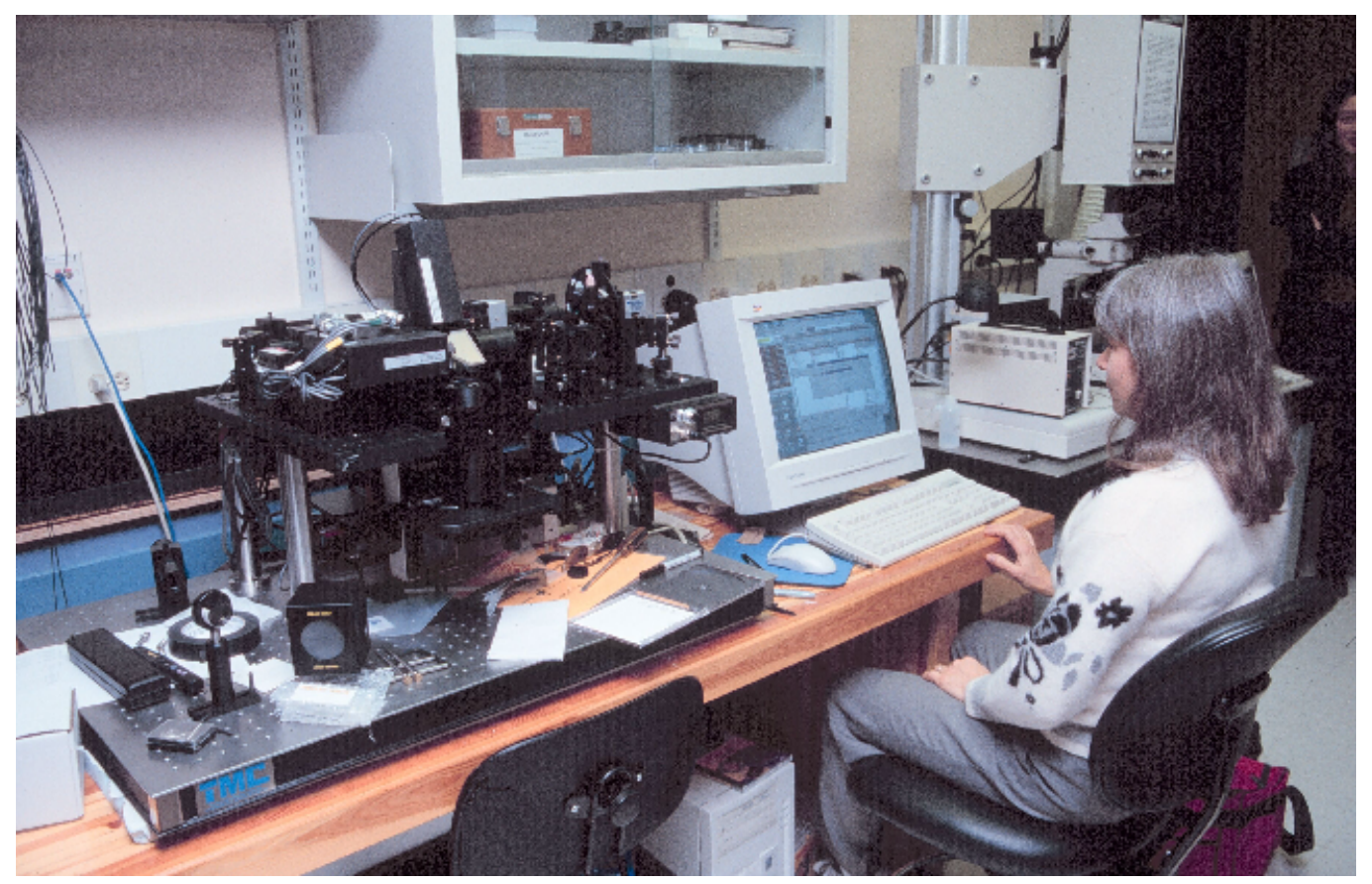

FIG. 1. Computer controlled MACROscope.

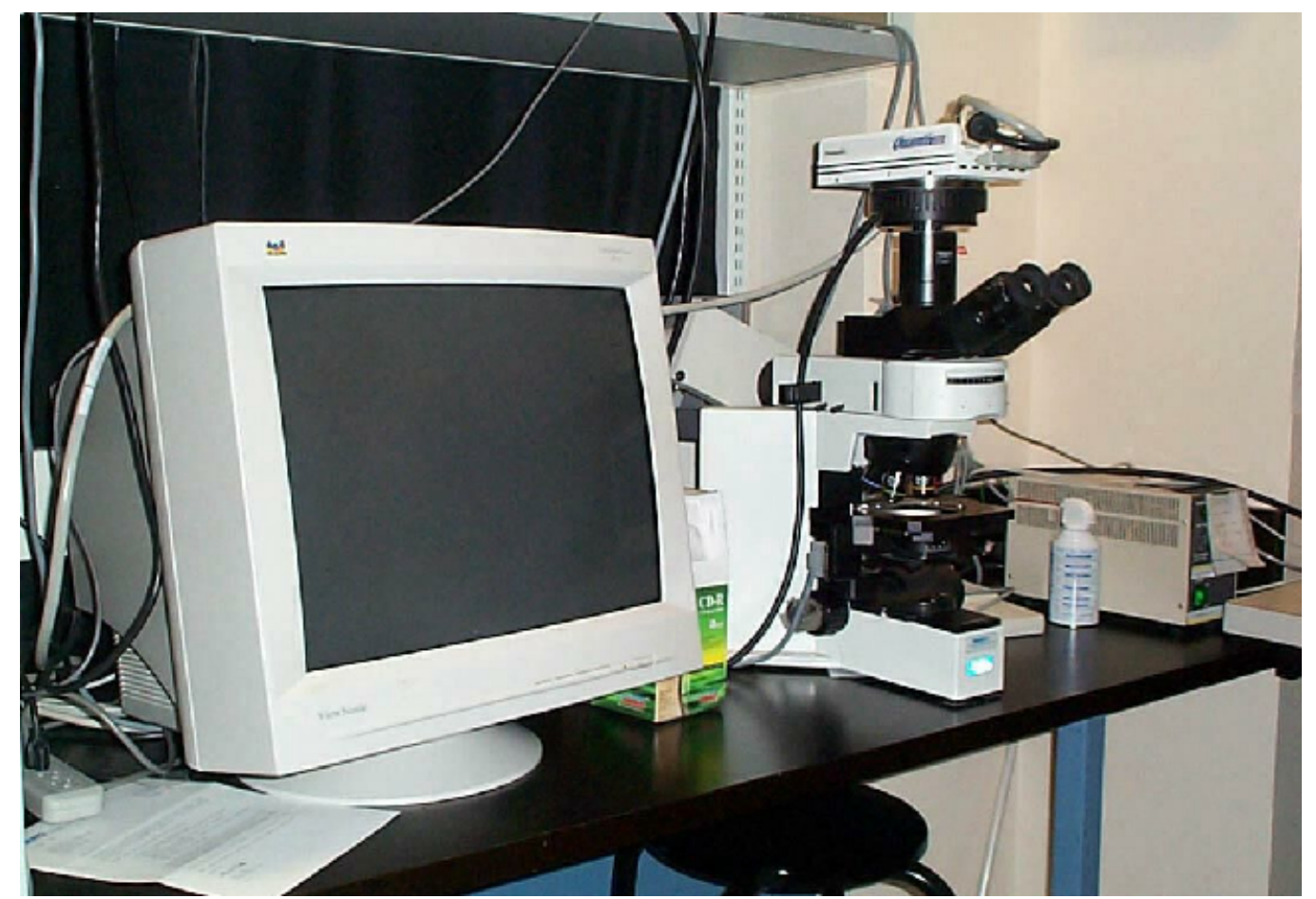

FIG. 2. Fluorescence Computerized Image Analysis system with motorized stage and cooled CCD camera. 
https://doi.org/10.1017/S1431927602107227 Published online by Cambridge University Press 